\title{
Dimensionamento de uma associação de bombas hidráulicas com uso de Arduino como parâmetro de automação
}

\author{
Alexsander Saves dos Santos ${ }^{1}$ \\ Caio Delano Couto Santos ${ }^{2}$ \\ Carlos Henrique Barbosa Sabino ${ }^{3}$ \\ Kauan Bernardes Cezar de Oliveira ${ }^{4}$
}

\begin{abstract}
RESUMO
O presente projeto teve como objetivo desenvolver mecanicamente um sistema de associações de bombas hidráulicas em paralelo e em série com características mistas, sendo controlado por mecanismo de automação como uso de Arduinos e relés. A metodologia utilizada foi uma pesquisa experimental, que permite avaliar o sistema hidráulico por meio da montagem da parte estrutural, elétrica, dos testes, coletas de dados e por métodos algébricos. O trabalho, portanto, permitiu a compreensão do sistema de associações de bombas hidráulicas, quando associadas em série apresenta uma altura manométrica de $2,16 \mathrm{~m}$ e uma vazão de $9,166 \times 10^{-5} \mathrm{~m}^{3} / \mathrm{s} \mathrm{e}$, quando associadas em paralelo, apresenta uma altura manométrica de $0,72 \mathrm{~m}$ e uma vazão de $1,83 \times 10^{-4} \mathrm{~m}^{3} / \mathrm{s}$, comprovando que o sistema em série gera maiores alturas manométricas, enquanto o sistema em paralelo promove maiores vazões volumétricas. Ademais, validou a eficiência da aplicação de um sistema com parâmetros automáticos.
\end{abstract}

Palavras-chave: Bombas. Associações Hidráulicas. Automação.

\footnotetext{
${ }^{1}$ Graduado em Física pelo Centro Universitário de Votuporanga - UNIFEV e em Pedagogia pela Faculdade da Aldeia de Carapicuíba - FALC. Mestre em Ciências Ambientais pela Universidade Brasil, ICESP. É professor nos Cursos de Engenharia Química e Medicina da Universidade Brasil, ICESP, Campus Fernandópolis, SP, Brasil. E-mail: saves.santos@gmail.com.

${ }^{2}$ Graduando em Engenharia Química pela Universidade Brasil, ICESP, Campus Fernandópolis, SP, Brasil. Email: caiodelanocolto@ hotmail.com.

${ }^{3}$ Graduando em Engenharia Química pela Universidade Brasil, ICESP, Campus Fernandópolis, SP, Brasil. Email: carlos18barbosa@outlook.com.

${ }^{4}$ Graduando em Engenharia Química pela Universidade Brasil, ICESP, Campus Fernandópolis, SP, Brasil. Email: kauanoliveira1313@hotmail.com.
} 


\title{
Sizing of association of hydraulic pumps with the use of the Arduino as auto-charge
}

\begin{abstract}
The present project aimed to mechanically develop a system of associations of hydraulic pumps in parallel and in series with mixed characteristics, the same being controlled by an automation mechanism such as the use of Arduino and relays. The methodology used was an experimental research, which allows the evaluation of the hydraulic system through the assembly of the structural, electrical, tests, data collections and algebraic methods. The work, therefore, allowed the understanding of the system of associations of hydraulic pumps, when associated in series presents a head of $2.16 \mathrm{~m}$ and a flow rate of $9.166 \times 10^{-5} \mathrm{~m}^{3} / \mathrm{s}$, when associated in parallel presents a head of $0.72 \mathrm{~m}$ and a flow of $1.83 \times 10^{-4} \mathrm{~m}^{3} / \mathrm{s}$, proving that the system in series generates greater head gauges, while the system in parallel promotes greater volumetric flows. Furthermore, it validated the efficiency of the application of a system with automatic parameters
\end{abstract}

Keywords: Pumps. Hydraulics Associations. Automation.

Artigo recebido em: 31/05/2020

Aceito em: 20/09/2020 


\section{INTRODUÇÃO}

Aproveitar o movimento do fluido tem sido fonte de energia durante anos (CREMASCO, 2014). Portanto, o estudo da mecânica dos fluidos em consonância com as operações unitárias promove o desenvolvimento e o aperfeiçoamento de novos conceitos e dispositivos que geram resultados para a sociedade.

"O artista e cientista Leonardo da Vinci (1452-1512), além dos seus outros trabalhos dedicou-se também a problemáticas hidráulicas" (NETTO, 1989). O artista incluiu em seus conhecidos desenhos concepções originais de máquinas hidráulicas, entre as quais a ideia das bombas hidráulicas.

Segundo Netto (1989), o século XVIII foi marcado por progressos na construção de bombas hidráulicas, como por exemplo, os de James Watt (1736-1819), que conseguiu melhorar consideravelmente o rendimento dos dispositivos hidráulicos, e os de Leonardo Euler (1707-1783), que analisou e desenvolveu princípios teóricos relacionado às bombas hidráulicas. Portanto, foi um período marcado por sucessivos aperfeiçoamentos que impulsionaram a industrialização. Desenvolvida por James Watt, a Figura 1 representa a primeira bomba hidráulica, movida a vapor.

Figura 1: Bomba hidráulica movida a vapor

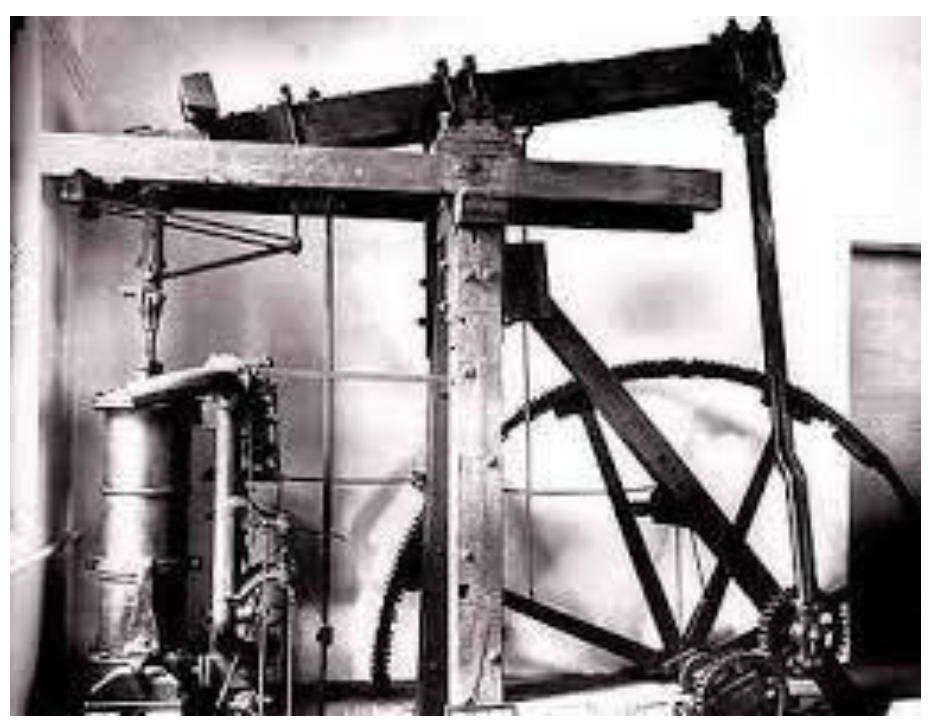

Fonte: Site Slideplayer (2019).

De acordo com a Eletrobrás (2009), as bombas hidráulicas, conhecidas como máquinas hidráulicas operatrizes, são amplamente utilizadas no setor industrial. Dessa forma, trata-se 
de um equipamento que permite o trabalho em conjunto com outros equipamentos, agregando e facilitando os processos industriais.

Segundo Cremasco (2014), as bombas hidráulicas podem ser definidas como um sistema hidráulico formado por componentes físicos que, quando associados, utilizam o fluido como meio de transferência de energia. As bombas são dispositivos mecânicos que recebem energia de uma fonte motora e cedem parte dessa energia ao fluido na forma de energia cinética, de energia de pressão, ou de ambas as formas (CREMASCO, 2014).

Existem vários modelos de bombas hidráulicas, que, de acordo com Terron (2012), podem ser divididas em dois grupos: bombas dinâmicas e bombas volumétricas. Podem ser utilizadas em bombeamento convencionais, abastecimentos de reservatórios e em sistemas de refrigeração, dentre outros.

O princípio fundamental das bombas dinâmicas é o aumento da energia cinética no propulsor e a conversão parcial em energia de pressão. Podem ser de quatro tipos: fluxo axial, com alta vazão e baixa pressão; fluxo misto, com pressão e vazão moderadas; regenerativas, com alta pressão e baixa vazão; e as centrífugas, com pressão e vazão altas (FOX; PRITCHARD; MCDONALD, 2006). A Figura 2 representa os tipos de impulsores das bombas dinâmicas:

Figura 2: Tipos de impulsores das bombas dinâmicas

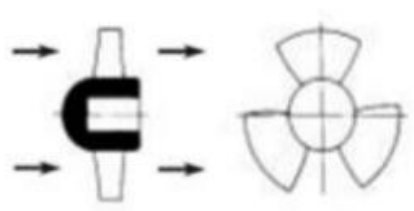

Axial

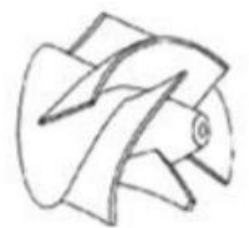

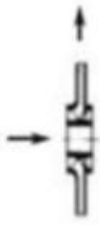

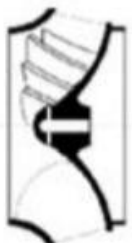

Tipo Francis

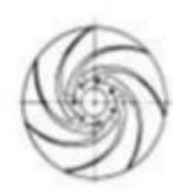

Radial

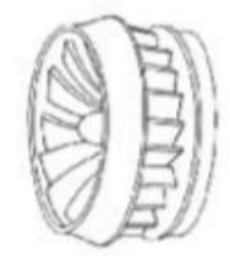

Regenerativa
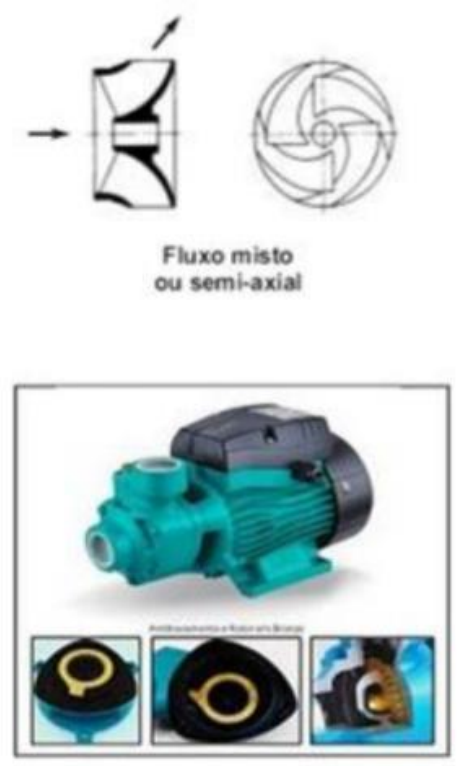

Fonte: Souza (2014). 
De acordo com Terron (2012), as bombas volumétricas, conhecidas como deslocamento positivo, ocorrem com a entrada e saída do fluido no interior da bomba de forma sucessiva, sendo que o movimento deste fluido dá-se na mesma direção das forças que são transmitidas ao mesmo. As bombas volumétricas podem ser divididas em: alternativas, como pistão, êmbolos e diafragmas, e rotativas, como engrenagens, lóbulos, palhetas, helicoidal, parafusos dentre outros (SOUZA, 2014). A Figura 3 demonstra exemplos de bombas volumétricas: (a) bomba de êmbolo; (b) bomba de engrenagem; (c) bomba helicoidal; (d) bomba palhetas; (e) bomba de lóbulos triplos; (f) bomba de pistão duplo e (g) bomba de rolete.

Figura 3: Exemplos de bombas volumétricas

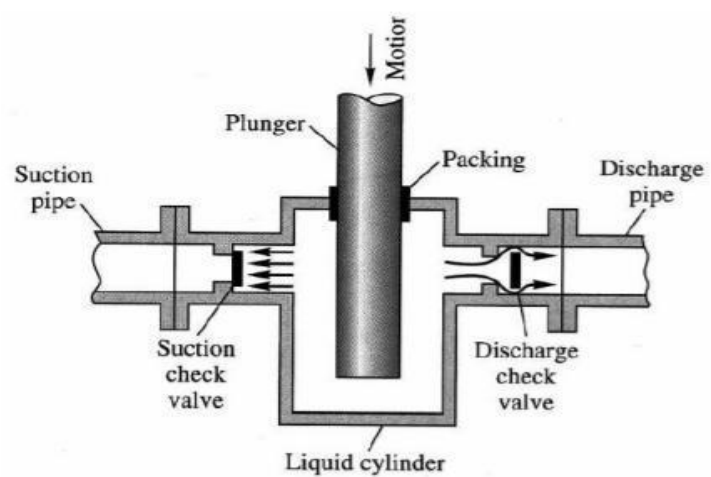

(a)

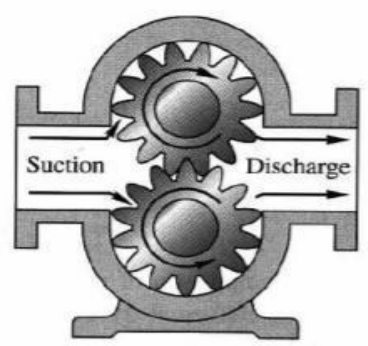

(b)

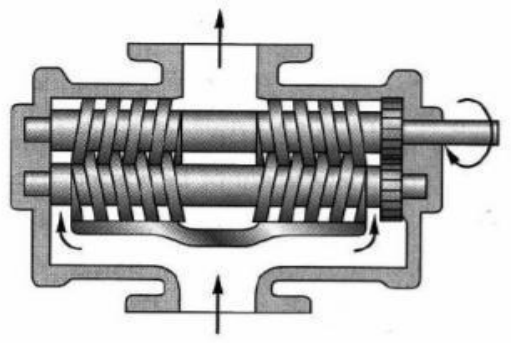

(c)

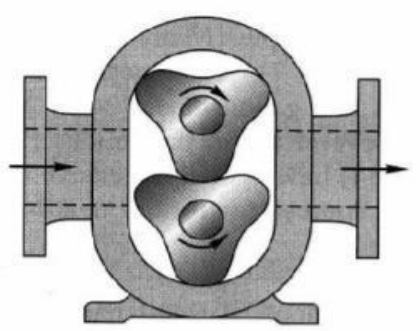

(e)

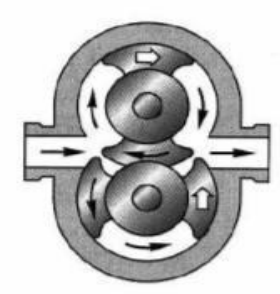

(f)

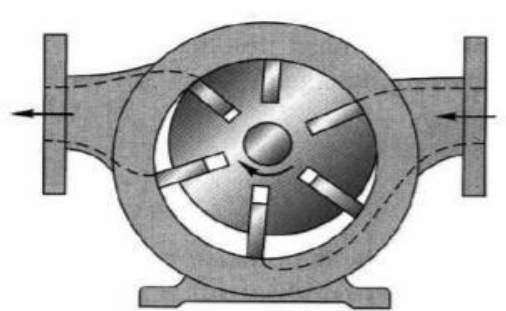

(d)

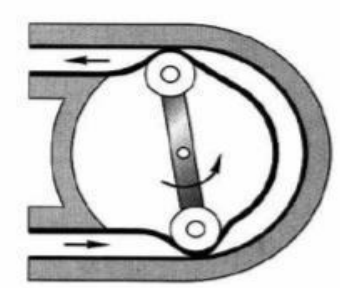

(g)

Fonte: Brasil (2010).

As bombas podem operar em associação em série, em paralelo, ou com características mistas. Segundo afirmações de Terron (2012), bombas associadas em série operam com a 
mesma vazão, porém fornecem alturas superiores do que produziriam isoladamente. Enquanto isso, a associação em paralelo é útil quando se precisam variar as vazões e manter a altura constante. Já as associações mistas são formadas por ambos os sistemas e possuem as mesmas propriedades. A Figura 4 representa a associação em paralelo e em série de duas bombas.

Figura 4: Configuração de duas bombas em associação em paralelo (acima) e em série (abaixo)
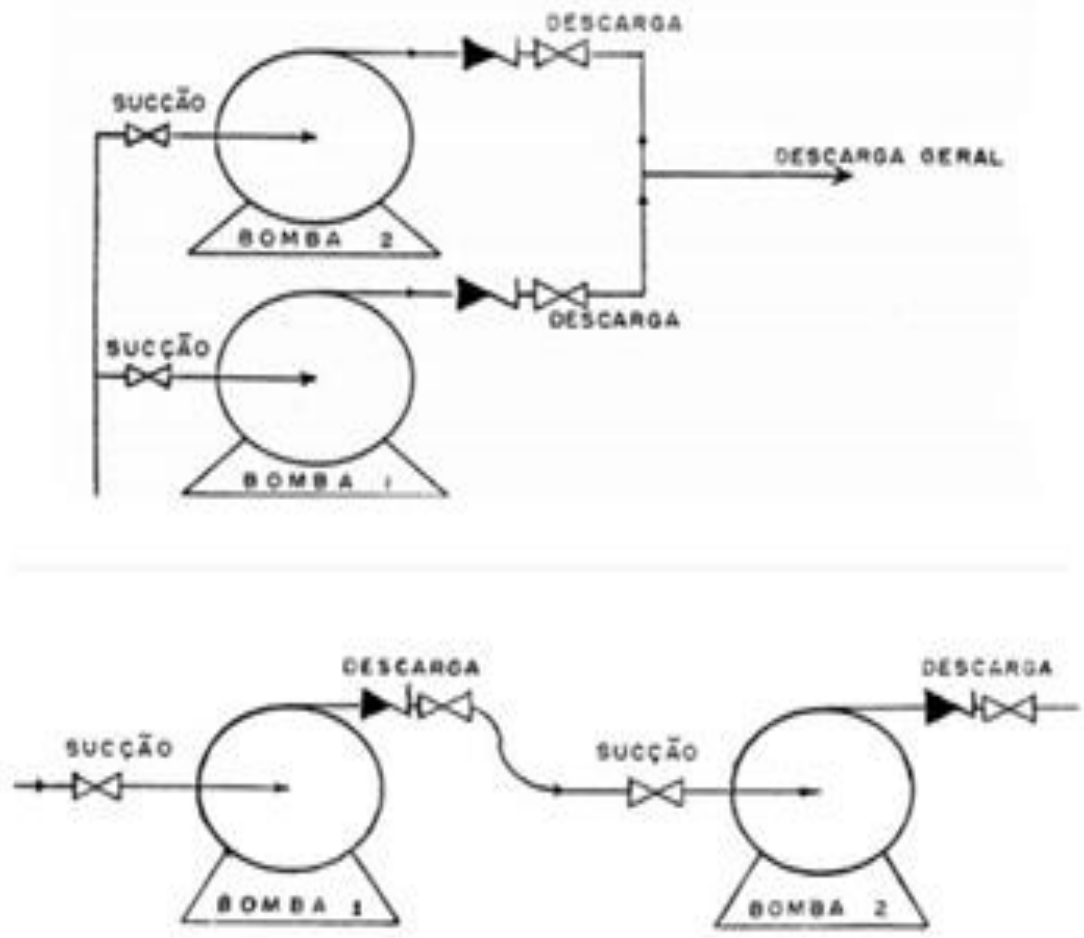

Fonte: Pinto (2013).

Um dos efeitos presentes no sistema de bombeamento é o atrito, o qual gera perda de carga que pode ser classificada em dois tipos: a localizada, que é ocasionada pelos obstáculos encontrados durante o percurso, tais como curvas e válvulas; e a distribuída, como o atrito em porções de área constante no sistema (FOX; PRITCHARD; MCDONALD, 2006)

De acordo com Gomes (2008), as associações de bombas hidráulicas possuem um papel fundamental nos processos industriais e possuem inúmeras aplicações, tais como processo de irrigação, sistemas de deslocamentos de produtos químicos e sistemas de condicionamento de ar. Elas facilitam os processos e geram lucros para a indústria.

Um sistema de bombas hidráulicas pode ser operado automaticamente. Conforme Gimenez (2005), o sistema automático é um meio moderno utilizado para controle dos 
mecanismos de funcionamento de equipamentos, garantindo a otimização de tempo, a redução de custos e o aumento da produtividade e da segurança.

Desenvolvido em 2005, por David Cuartielles e Massimo Banzi, o Arduino é uma plataforma de prototipagem que promove um sistema automático. É constituído por uma placa com microcontrolador e uma IDE- Ambiente de Desenvolvimento Integrado (do inglês, Integrated Development Environment) cuja linguagem é baseada em $\mathrm{C} / \mathrm{C}++$ (FELIPEFLOP, 2018). Com isso, o Arduino pode ser visto como um dispositivo simples que permite ao usuário desenvolver inúmeras programações. A Figura 5 representa a estrutura da placa de Arduino Uno.

Figura 5: Placa de Arduino do tipo Uno

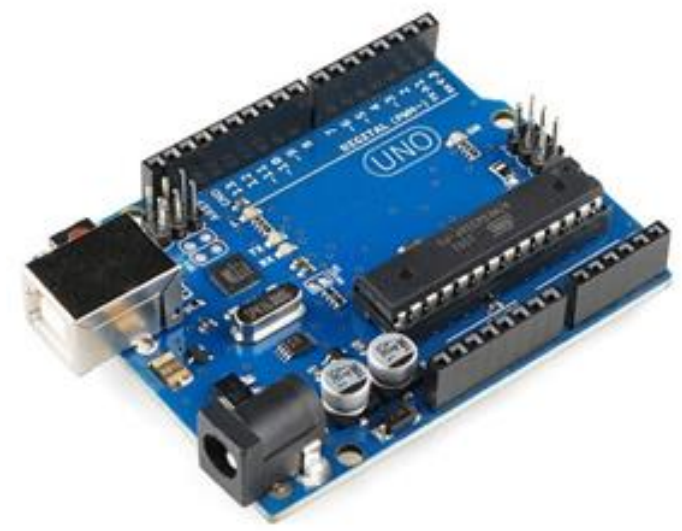

Fonte: Site Felipeflop (2018).

O Arduino possibilita inúmeras ideias e é de extrema importância para a sociedade. Pode ser utilizado em atividades temporizadas, na interação com ambiente, no controle de temperatura e luminosidade, dentre outras possibilidades (FRIZZARIN, 2016).

O presente trabalho, portanto, visa desenvolver mecanicamente um sistema de associações de bombas hidráulicas em paralelo e em série, com o uso de Arduino como mecanismo de automação.

\section{METODOLOGIA}

O presente projeto foi realizado na Universidade Brasil em Fernandópolis-SP, situada na Estrada F-1, s/n, Fazenda Santa Rita, entre os meses de agosto e dezembro de 2019.

Com efeito, foi realizada uma pesquisa de caráter experimental, cuja finalidade foi apresentar uma associação de bombas com características de automação. Com isso, visando 
uma melhor compreensão da metodologia empregada, foi desenvolvido um itinerário no formato de fluxograma, representado pela Figura 6.

Figura 6: Fluxograma do mecanismo empregado na metodologia

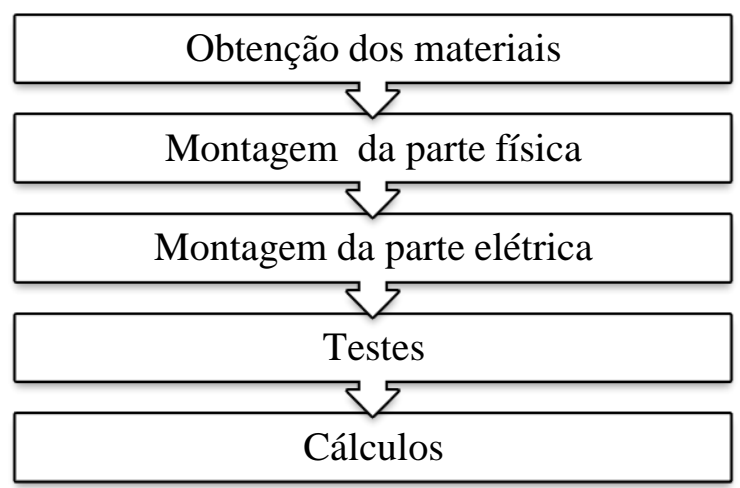

Fonte: Autoria própria.

\subsection{Montagem do sistema de associação de bombas hidráulicas}

Os materiais foram adquiridos com recursos próprios a partir da parceria com uma empresa de vidraçaria da cidade de Iturama - MG. Seguem, portanto, pormenorizados todos os itens utilizados na prática experimental, conforme Quadro 1 e Quadro 2.

Quadro 1: Materiais referentes à unidade estrutural.

\begin{tabular}{|c|c|c|}
\hline Materiais & Unidade & Quantidade \\
\hline Reservatórios de Vidro & un & 39 \\
\hline Abraçadeira borboleta & un & 30 \\
\hline Abraçadeira tipo U $1 / 2$ & un & 12 \\
\hline Terminal Fêmea & un & 11 \\
\hline Joelho interno duplo & un & 1 \\
\hline T interno $1 / 2$ & un & 2 \\
\hline Adaptador interno $1 / 2$ & un & 5 \\
\hline Joelho interno $1 / 2$ & un & 5 \\
\hline Adap. Flange & un & 4 \\
\hline Mangueira cristal & m & 60 \\
\hline Parafuso Phillips & un & \\
\hline
\end{tabular}

Continua... 
... Conclusão

\begin{tabular}{|c|c|c|}
\hline Materiais & Unidade & Quantidade \\
\hline Joelho com rosca $90^{\circ}$ & un & 1 \\
\hline Bicos de torneira metal & un & 3 \\
\hline Mangueira corrugada & m & 6 \\
\hline Spray preto & un & 2 \\
\hline Silicone selamais & un & 1 \\
\hline Cantoneira mão francesa & un & 4 \\
\hline
\end{tabular}

Fonte: Autoria própria.

Quadro 2: Materiais referentes à unidade elétrica

\begin{tabular}{|c|c|c|}
\hline Materiais & Unidade & Quantidade \\
\hline Bomba de diafragma & un & 3 \\
\hline Relé 4 canais & un & 2 \\
\hline Válvula solenóide & un & 4 \\
\hline Placa de Arduíno Uno & un & 2 \\
\hline Fonte de 127V para 12V & un & 1 \\
\hline Terminal ilhós & un & 15 \\
\hline Cabo PP 2x1 & m & 9 \\
\hline Válvula dupla enxuta & un & 2 \\
\hline
\end{tabular}

Fonte: Autoria própria.

A Figura 7 apresenta o desenho esquemático do projeto do sistema de associação de bombas hidráulicas elaborado por meio de uma versão do Autodesk. O esquema destaca as posições das bombas hidráulicas, das válvulas solenóides, dos reservatórios e das tubulações. 
Figura 7: Desenho esquemático do sistema de associação de bombas

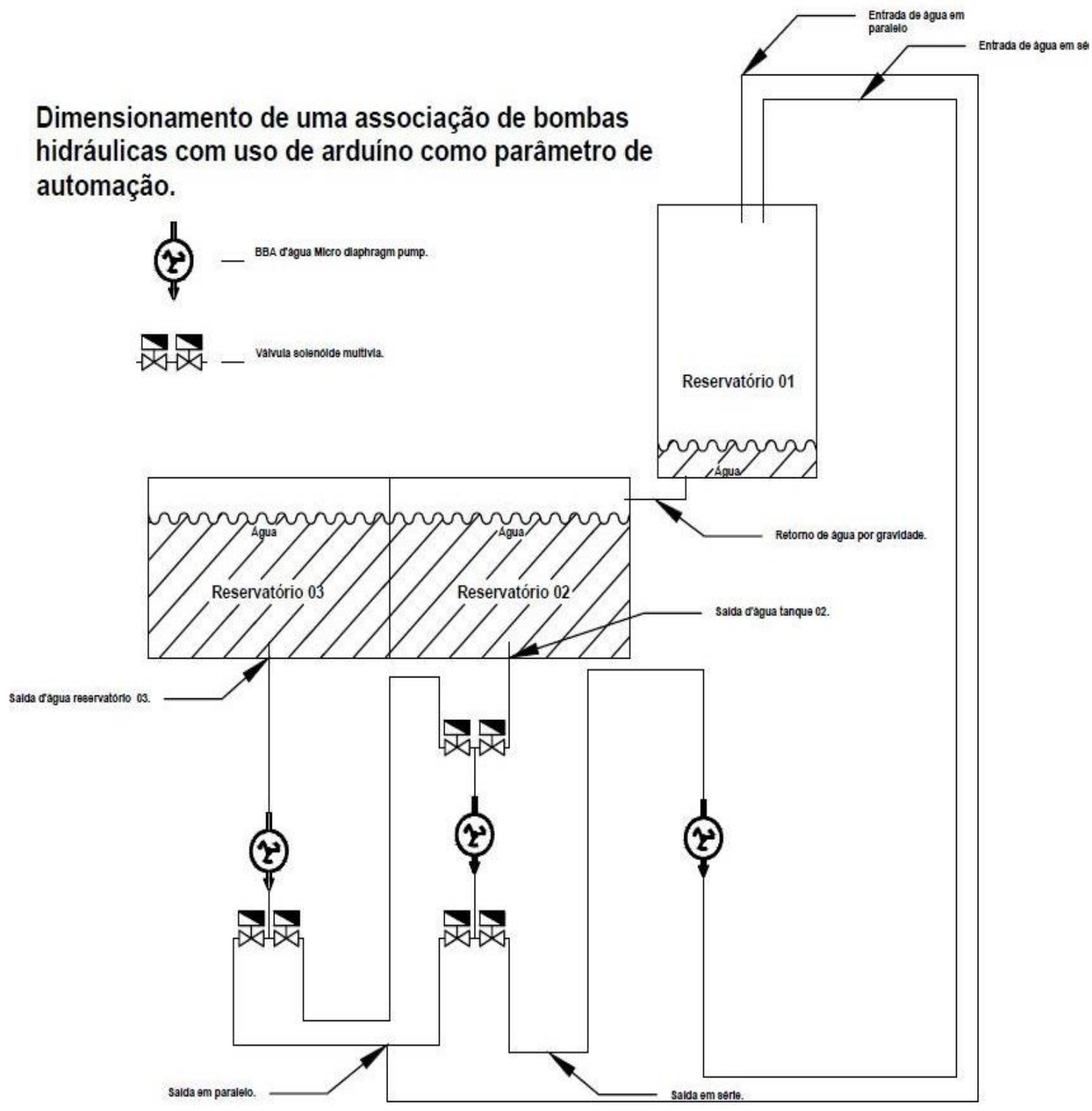

Fonte: Autoria própria.

A montagem da unidade estrutural consistiu em três reservatórios de capacidade máxima de 16 litros cada, com abertura no topo e flange para saída de água na base. Esses tanques alimentam os sistemas formados por mangueira cristal e conectados por conexões em $\mathrm{T}$ e joelhos de $90^{\circ}$. Nesse sistema, há três bombas de diafragma de $12 \mathrm{~V}$ instaladas, as quais aspiram a água alimentada pelo reservatório, bombeando-a de volta para o mesmo. Foram instalados três solenóides em posições estratégicas para que, dependendo da combinação de abertura ou fechamento delas, fosse possível operar cada uma das bombas isoladamente ou associadas em série, em paralelo ou caráter misto. 
O controle de todo o sistema foi feito por meio de dois relés de quatro vias, dispositivo cuja função está no controle de abertura e fechamento dos solenóides. Os relés são controlados pelas placas de Arduino com vista às adaptações elaboradas por um software, neste caso um Sketch. O Quadro 3 mostra as conexões entre as placas de Arduino e os módulos de relés, visando o controle do sistema hidráulico.

Quadro 3: Conexões digitais entre relés e placas de Arduino

\begin{tabular}{|c|c|}
\hline Relé $\mathbf{4}$ vias & Arduino \\
\hline $\mathrm{Dc}+/ \mathrm{Vcc} / 5 \mathrm{~V}$ & $5 \mathrm{~V}$ \\
\hline $\mathrm{GND}$ & GND \\
\hline $\mathrm{IN}$ & Portal digital \\
\hline
\end{tabular}

Fonte: Autoria própria.

As principais etapas de montagem da parte estrutural e elétrica podem ser mais bem visualizadas na Figura 8.

Figura 8: Principais etapas de montagem
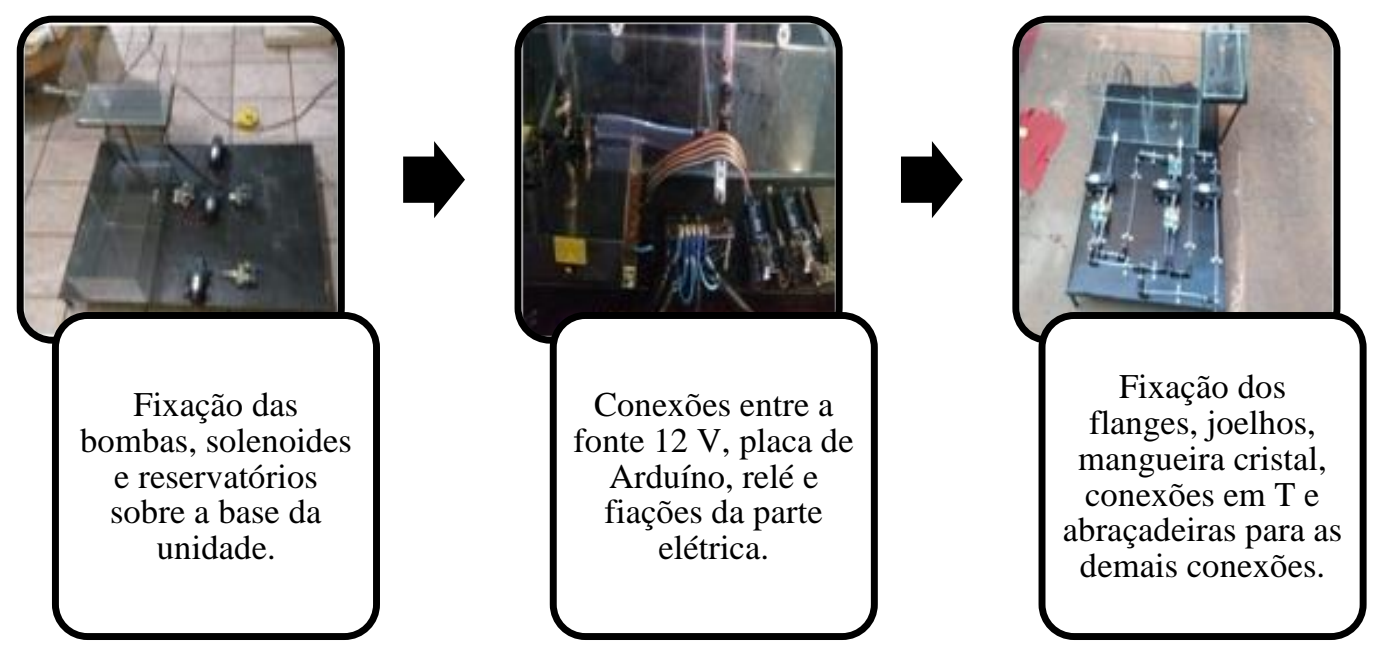

Fonte: Autoria própria.

Em seguida, desenvolveu-se a linguagem de programação para o sistema operar em série e em paralelo. Definiu-se um conjunto de instruções na forma de Sketch, através do próprio Arduino. Essa programação foi realizada utilizando um computador, cabo USB e um ambiente de programação chamado de IDE - Ambiente de Desenvolvimento Integrado (do inglês, Integrated Development Environment). Criou-se o texto de programação com as instruções necessárias para controlar as bombas e tempo de abertura e fechamento dos solenóides e, assim, definir o sistema automático. As Figuras 9 e 10 representam o texto de 
programação finalizado utilizado no projeto, referindo-se às associações em série e em paralelo respectivamente.

Figura 9: Texto de programação finalizado da associação de bombas em série

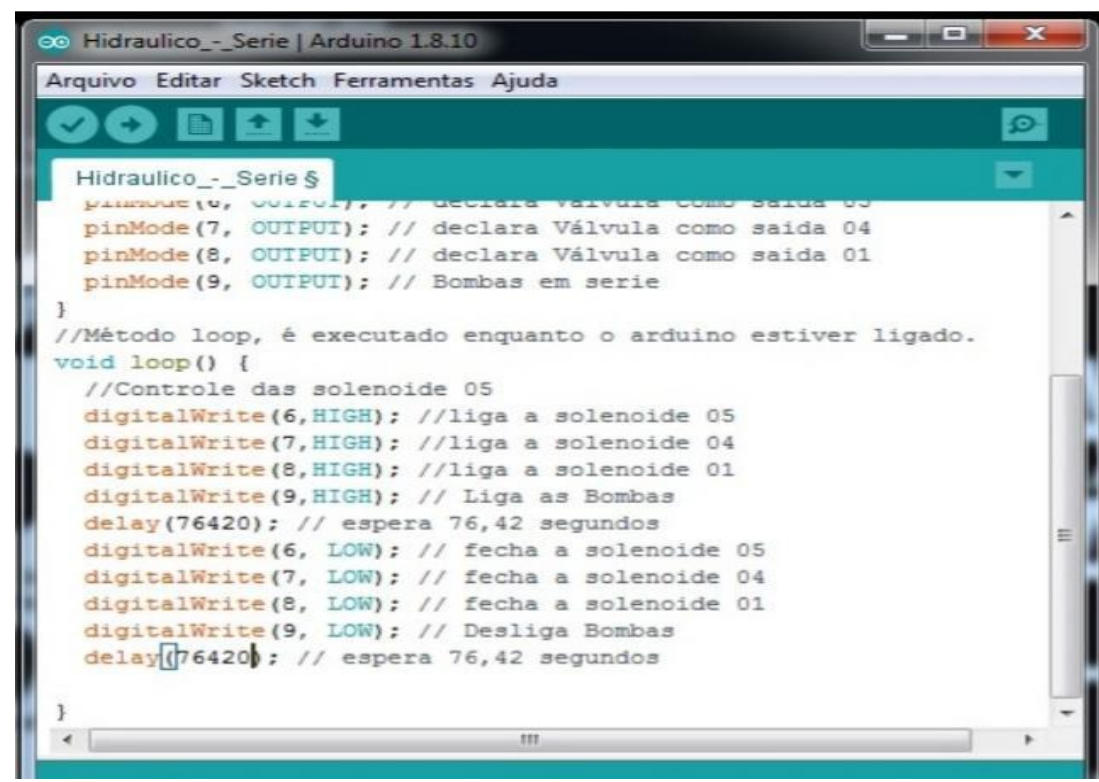

Fonte: Autoria própria.

Figura 10: Texto de programação finalizado da associação em paralelo

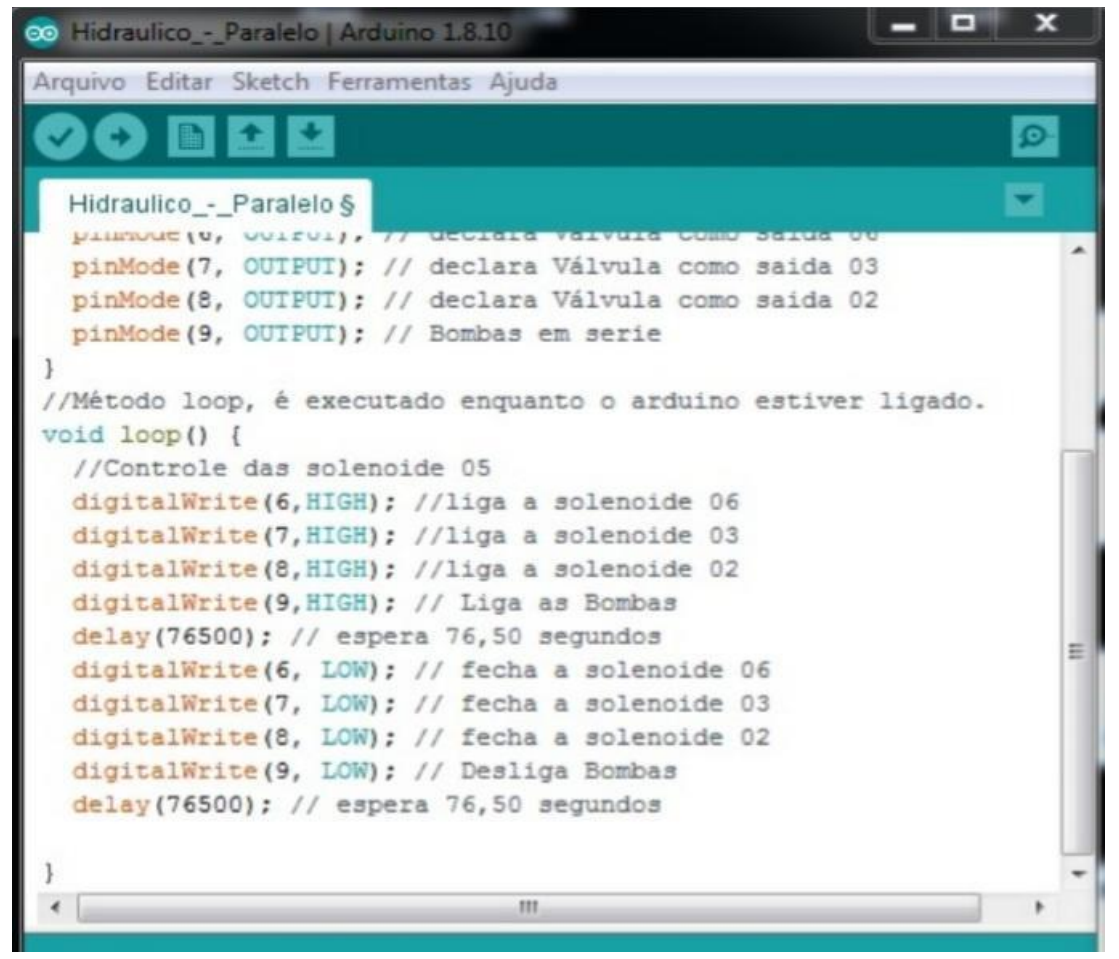

Fonte: Autoria própria. 
Com o objetivo de controlar o fluxo do líquido, instalou-se no projeto válvulas solenóides, que trabalham em dois estágios: totalmente aberto ou totalmente fechado. $\mathrm{O}$ Quadro 4 representa os estágios das válvulas solenóides durante o funcionamento do sistema quando associadas em paralelo e em série.

Quadro 4: Estágios das válvulas solenóides dos sistemas em associações

\begin{tabular}{|c|c|c|}
\hline Válvulas solenóides & Associação em paralelo & Associação em série \\
\hline 01 & Fechado & Aberto \\
\hline 02 & Aberto & Fechado \\
\hline 03 & Aberto & Fechado \\
\hline 04 & Fechado & Aberto \\
\hline 05 & Fechado & Aberto \\
\hline 06 & Aberto & Fechado \\
\hline
\end{tabular}

Fonte: Autoria própria.

A Figura 11 demonstra o projeto de associações de bombas hidráulicas em paralelo e em série finalizadas.

Figura 11: Sistema de associação de bombas concluída

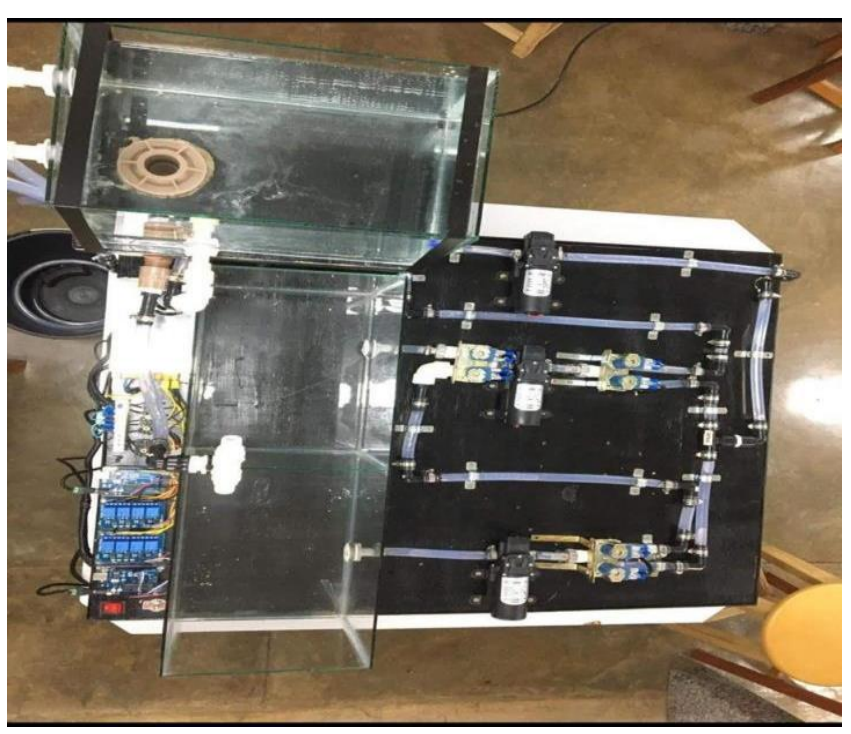

Fonte: Autoria própria.

\subsection{Teste do sistema de associação de bombas hidráulicas}


Buscando verificar o funcionamento do sistema, foram realizados três testes preliminares. Para isso, elaborou-se um roteiro constituído por duas fases: preparação e execução.

A primeira, a preparação, consistiu em encher os reservatórios inferiores com volumes de 10 L, 12 L, 14 L e 16 L de água, respectivamente, e verificar o volume suficiente para preenchimento de toda a linha de escoamento tanto em série quanto em paralelo. Em seguida, verificou-se a normalidade de funcionamento de todos os equipamentos presentes na unidade, como bombas, solenóides e painel de controle.

Na segunda fase, a execução, ligaram-se as bombas por meio de um painel de controle, analisou-se o comportamento do sistema trabalhando em associação em série e em paralelo e observou-se a ocorrência de ajustes de projeto.

\subsection{Métodos analíticos do sistema de associação de bombas hidráulicas}

Aplicaram-se métodos algébricos com o propósito de avaliar o desempenho do projeto e de compreender as diferenças de comportamento das bombas associadas em série e em paralelo. Para a obtenção da velocidade, utilizou-se a equação:

$$
v=\frac{Q}{A}
$$

onde $v$ é a velocidade dada em $\mathrm{m} / \mathrm{s}, Q$ é a vazão em $\mathrm{m}^{3} / \mathrm{s}$ e $A$ é a área da tubulação $\mathrm{em}^{2}$.

Para a classificação do regime de escoamento, foi aplicada a Equação 2 desenvolvida pelo físico Osborne Reynolds em 1883:

$$
R_{e}=\frac{\rho v D}{\mu}
$$

Onde $R_{e}$ é o número de Reynolds e é adimensional, $v$ é a velocidade em $\mathrm{m} / \mathrm{s}, \rho$ é a massa específica em $\mathrm{kg} / \mathrm{m}^{3}, D$ é o diâmetro medido em metros, e $\mu$ é o coeficiente de viscosidade do fluído em $\mathrm{m}^{2} / \mathrm{s}$.

Ao classificar o comportamento do escoamento, escolheu-se o método de Haaland, pois, segundo Çengel e Cimbala (2007), ele permite o cálculo do coeficiente de atrito, uma propriedade intrínseca da perda de carga, cuja fórmula é apresentada na Equação 3: 


$$
\frac{1}{\sqrt{f}}=-1,8 \log \left(\frac{6,9}{R e}+\left(\frac{\frac{E}{D}}{3,7}\right)^{1,11}\right)
$$

em que $f$ é o coeficiente de atrito e é adimensional, $R_{e}$ é o número de Reynolds (adimensional), $E$ é a constante de rugosidade, neste caso da mangueira cristal dada em metros, e $D$ é o diâmetro da tubulação medido em metros.

Para obtenção da perda de carga, foi utilizado o método de Darcy-Weisbach aplicado a qualquer tipo de fluido incompreensível e a tubulações de qualquer diâmetro e material. Dessa forma, sua expressão é representada pela Equação 4:

$$
h_{p}=f \frac{L v^{2}}{D 2 g}
$$

onde $h_{p}$ é a perda de carga em $\mathrm{m}, f$ é o coeficiente de atrito (adimensional), $L$ é o comprimento em $\mathrm{m}, v$ é a velocidade em $\mathrm{m} / \mathrm{s}, D$ é o diâmetro da tubulação em $\mathrm{m}$ e $g$ é a força gravitacional em $\mathrm{m} / \mathrm{s}^{2}$.

Para o cálculo da altura manométrica, foi utilizada a equação da energia desenvolvida por Bernoulli:

$$
H_{\text {man }}=\frac{P_{2}-P_{1}}{\gamma}+\frac{v_{2}^{2}-v_{1}^{2}}{2 g}+z_{2}-z_{1}+h_{p}
$$

em que $P$ é a pressão medida em $\mathrm{Pa}\left(=\mathrm{N} / \mathrm{m}^{2}\right), v$ é a velocidade em $\mathrm{m} / \mathrm{s}$, $\gamma$ é o peso específico em $\mathrm{N} / \mathrm{m}^{3}, z$ é a cota/altura em $\mathrm{m}, g$ é a força gravitacional dada em $\mathrm{m} / \mathrm{s}^{2}, h_{p}$ é a perda da carga em m, e $H_{\text {man }}$ é a altura manométrica em metros.

Ao final, utilizou-se a Equação 6 para o cálculo do tempo de escoamento do fluido durante todo o percurso, tanto em série quanto em paralelo:

$$
t=\frac{V}{Q}
$$

onde $t$ é o tempo dado em $\mathrm{s}, V$ é o volume em $\mathrm{m}^{3}$ e o $Q$ é a vazão $\mathrm{em}^{3} / \mathrm{s}$.

\section{RESULTADOS E DISCUSSÃO}

O projeto de associação de bombas hidráulicas busca entender o comportamento do sistema quando associadas em série e em paralelo, sendo controlado por um sistema automático com aplicação de microcontroladores (Arduinos). 
Primeiramente, houve dificuldade durante a montagem estrutural da unidade devido a ocorrências de vazamentos de fluido entre as conexões das tubulações. A fim de corrigir o problema, aumentou-se a quantidade de abraçadeiras para a vedação das mangueiras, evitando assim novos vazamentos.

O tempo programado no Arduino para o funcionamento do projeto foi de aproximadamente $76 \mathrm{~s}$ para operar em associação em paralelo e $152 \mathrm{~s}$ para associação em série. Como ambos trabalham com o mesmo volume e a vazão do sistema associado em paralelo é o dobro comparado com o sistema em série, o tempo, portanto, do sistema em série será o dobro em relação ao do sistema em paralelo. Após a realização dos testes, calculou-se qual o volume ideal para um bom funcionamento nos reservatórios inferiores, obtendo um volume de 14 litros de água, quantidade essa para preencher toda a linha de escoamento (tubulação) de forma que todos os dispositivos funcionem no tempo programado. O Quadro 5 apresenta os resultados dos testes dos volumes.

Quadro 5: Resultados dos testes

\begin{tabular}{|c|c|c|c|c|}
\hline Associação & Volume 10 L & Volume 12 L & Volume 14 L & Volume 16 L \\
\hline \begin{tabular}{c} 
Tempo em série \\
\hline $\begin{array}{c}\text { Tempo em } \\
\text { paralelo }\end{array}$
\end{tabular} & $54,64 \mathrm{~s}$ & $65,57 \mathrm{~s}$ & $76,52,73 \mathrm{~s}$ & $174,55 \mathrm{~s}$ \\
\hline
\end{tabular}

Fonte: Autoria própria.

Visando obter resultados por meio dos métodos analíticos, utilizaram-se as informações da bomba de diafragma, em que se indica uma tensão de $12 \mathrm{~V}$, uma potência de $80 \mathrm{~W}$, pressão de trabalho de 130 psi, uma vazão máxima é de 5,5 L/min, ou seja, 330 litros por hora, apresentam uma sucção máxima de $2 \mathrm{~m}$ e uma elevação máxima de $40 \mathrm{~m}$ (MERCADO LIVRE, 2020).

A fim de avaliar o desempenho do projeto, compararam-se os resultados dos cálculos e do sistema automático com as literaturas cujo desenvolvimento é semelhante ao projeto de associações de bombas com parâmetros de automação.

De acordo com Caixeta (1991), em seus estudos sobre perdas de cargas em tubos e conexões de PVC utilizados em sistemas portáteis de irrigação presentes em um sistema de bombeamento hidráulico, a vazão de uma bomba é de $1,6 \times 10^{-3} \mathrm{~m}^{3} / \mathrm{s}$. A bomba utilizada no projeto possui uma vazão de $9,166 \times 10^{-5} \mathrm{~m}^{3} / \mathrm{s}$. 
Inicialmente, os cálculos foram desenvolvidos buscando-se entender, por meio dos métodos algébricos, o comportamento de uma única bomba. Com o valor do diâmetro da tubulação de meia polegada, calculou-se a área da tubulação e, com o valor da vazão de $9,166 \times 10^{-5} \mathrm{~m}^{3} / \mathrm{s}$, obteve-se uma velocidade de $0,81 \mathrm{~m} / \mathrm{s}$. De acordo com Caixeta (1991), uma tubulação de PVC com vazão $1,6 \times 10^{-3} \mathrm{~m}^{3} / \mathrm{s}$ promoveu uma velocidade de $0,93 \mathrm{~m} / \mathrm{s}$.

Com o valor da massa específica e do coeficiente de viscosidade da água, obteve-se o número de Reynolds igual a 9690,9, confirmando um regime de escoamento turbulento. De acordo com Cremasco (2014) e com a Associação Brasileira de Normas Técnicas - ABNT, o regime de escoamento da água pode ser classificado como turbulento quando seu valor é superior a 4000. Segundo Caixeta (1991), em seu estudo, o número de Reynolds obtido considerando uma velocidade de $0,93 \mathrm{~m} / \mathrm{s}$ foi de 42690, o regime de escoamento é classificado como turbulento.

Com os valores do número de Reynolds, diâmetro da tubulação e da constante de rugosidade da mangueira cristal, utilizou-se o método de Haaland e se obteve o valor do coeficiente de atrito de 0,0338. Segundo Caixeta (1991), o coeficiente de atrito de uma tubulação de PVC com uma vazão de $1,6 \times 10^{-3} \mathrm{~m}^{3} / \mathrm{s}$ e com uma velocidade de $0,93 \mathrm{~m} / \mathrm{s}$ foi de 0,0220 .

Observa-se que o efeito do atrito gera perda, seja ela localizada ou distribuída. Com isso, neste projeto identificou-se em uma perda de carga igual a 0,377 m. Conforme Caixeta (1991), por meio da equação Darcy-Weisbach, a perda de carga foi de 0,3234 m.

Depois de obtidas todas as variáveis necessárias, como velocidade, número de Reynolds, coeficiente de atrito e perdas de carga, encontrou-se a altura manométrica referente a uma única bomba de $0,72 \mathrm{~m}$.

Após compreender o comportamento de uma bomba por meio dos métodos algébricos, calculou-se a vazão, a velocidade, a altura manométrica e o tempo de escoamento nas associações, considerando-se três bombas trabalhando em série e duas bombas trabalhando em paralelo. O Quadro 6 demonstra os cálculos dos sistemas em associações. 
Quadro 6: Resultados dos cálculos das associações de bombas

\begin{tabular}{|c|c|c|c|c|}
\hline Associação & Vazão $\left(\mathbf{m}^{3} / \mathbf{s}\right)$ & Velocidade $(\mathbf{m} / \mathbf{s})$ & $\begin{array}{c}\text { Altura } \\
\text { manométrica } \\
(\mathbf{m})\end{array}$ & \multicolumn{1}{|c|}{ Tempo (s) } \\
\hline $\begin{array}{c}\text { Série: } \\
\text { bombas }\end{array}$ & $9,166 \times 10^{-5}$ & $\frac{9,166 \times 10^{-5}}{\frac{\pi \times 0,012^{2}}{4}}$ & $3 \times 0,72=2,16$ & $\begin{array}{l}\frac{14 \times 10^{-3}}{9,166 \times 10^{-5}} \\
=152,7\end{array}$ \\
\hline $\begin{array}{c}\text { Paralelo: } \\
2 \text { bombas }\end{array}$ & $\begin{array}{l}2 \times 9,166 \times 10^{-5} \\
=1,83 \times 10^{-4}\end{array}$ & $\frac{1,83 \times 10^{-4}}{\frac{\pi \times 0,012^{2}}{4}}=1,62$ & 0,72 & $\frac{14 \times 10^{-3}}{1,83 \times 10^{-4}}=76,5$ \\
\hline
\end{tabular}

Fonte: Autoria própria.

Ao analisar os valores da associação em série de três bombas iguais e com instalações similares, observa-se que trabalham com a mesma vazão, enquanto a altura manométrica é definida pela contribuição de cada bomba (CARNEIRO; CRUZ, 2018). Dessa forma, a associação em série possui altura manométrica superior em relação à associação em paralelo, porém leva mais tempo para escoar um volume de 14 litros de água do reservatório.

No caso da associação em paralelo de duas bombas iguais e com instalações similares, segundo Carneiro e Cruz (2018), essa associação requer um aumento da vazão, porém as bombas trabalham com a mesma altura manométrica, sendo que, no caso de falha em uma das bombas, haverá apenas uma diminuição da vazão. Com isso, a associação em paralelo possui vazão superior em relação às associações em série, ademais, demonstrou ser mais rápida em escoar um volume de 14 litros de água do reservatório.

Segundo Queiroz e Souza (2018), é de extrema importância desenvolver ou adaptar novos meios em projetos experimentais. Dessa forma, como o Arduino é um dispositivo que permite o funcionamento de forma independente, a sua aplicação no projeto gera um ação automática, promovendo uma otimização do sistema hidráulico.

De acordo com Queiroz e Souza (2018), adeptos dos conceitos de Frizzarin (2016), a principal restrição do uso do Arduino é que se trata de uma plataforma já modelada e, por isso, encontra-se dificuldade caso se queira mudar de tipo de arquitetura. Porém, durante o desenvolvimento do projeto, o Arduino proporcionou um conjunto de ferramentas e sensores que facilita a interação com outros dispositivos, como por exemplo, com as bombas hidráulicas e solenóides. 
De acordo com Frizzarin (2016), o sistema automático é útil na substituição de um sistema manual, pois promove precisão, agilidade e lucro. O Arduino, portanto, demonstrou ser útil na automatização do processo de associação de bombas hidráulicas.

\section{CONCLUSÃO}

Nota-se que o projeto permitiu compreender o comportamento do sistema de bombas hidráulicas em associação tanto em série quanto em paralelo. Conseguiu-se avaliar o desempenho do projeto por meio dos métodos algébricos, obtendo-se resultados coerentes com a literatura.

Ao realizar os cálculos referentes a uma bomba utilizando-se métodos como os de Osborne Reynolds, Haaland e de Darcy-Weisbach, foi possível classificar o regime de escoamento do projeto como turbulento e obtiveram-se valores de variáveis tais como o do coeficiente de atrito de 0,0338 e de uma perda de carga de $0,377 \mathrm{~m}$, o que permitiu os cálculos para associação.

Com isso, conseguiu-se comprovar que a associação de três bombas em série promove uma altura manométrica de 2,16 m, sendo esta superior à da associação em paralelo, com uma altura de $0,72 \mathrm{~m}$. Porém, a associação de duas bombas em paralelo proporciona uma vazão de $1,83 \times 10^{-4} \mathrm{~m}^{3} / \mathrm{s}$, sendo esta superior em relação à associação em série, com uma vazão de $9,166 \times 10^{-5} \mathrm{~m}^{3} / \mathrm{s}$.

O trabalho conseguiu validar o sistema automático como um método de controle ideal tendo como referência o sistema manual, mesmo com restrições; comprovou que o sistema automático com o uso de Arduinos é eficaz devido a sua facilidade de interação com outros dispositivos.

\section{REFERÊNCIAS}

BRASIL, Alex, N. Bombas - Classificação e descrição, 2010. Disponível em: https://sistemas.eel.usp.br/docentes/arquivos/5817712/LOQ4015/capitulo3_bombasclassifi cacaoedescricao.pdf. Acesso em: 16 jul. 2020.

CAIXETA, Anicésio Vieira. Perdas de cargas em tubos e conexões de PVC utilizados em sistemas portáteis de irrigação por aspersão. 1991. 115 f. Dissertação (Mestrado em Agronomia) - Universidade de São Paulo, Piracicaba/SP, 1991. Disponível em: 
http://www.teses.usp.br/teses/disponiveis/11/11143/tde-20181127-

155322/publico/CaixetaAnicesioVieira.pdf. Acesso em: 17 jul 2020.

CARNEIRO, Vinicius; CRUZ, Vaguinelson. Associação de bombas em série e paralelo. Macapá - AP, 2018.

CREMASCO, Marco Aurélio. Operações unitárias em sistemas particulados e fluidomecânicos. 2. ed. São Paulo: Blucher, 2014.

ÇENGEL, Y. A.; CIMBALA, J. M. Mecânica dos Fluidos - Fundamentos e Aplicações. 1. ed. São Paulo: McGraw-Hill Companies, 2007.

ELETROBRÁS - Centrais Elétricas Brasileiras S.A (et al.). Bombas: Guia Básico. Brasília: Instituto Euvaldo Lodi - IEL/Núcleo Central-NC, 2009. p. 15-158.

FELIPEFLOP, Componentes Eletrônicos, 2018. Disponível em: https://www.filipeflop.com/. Acesso em: 13 jul. 2020.

FOX, R.W.; PRITCHARD, P.J.; MCDONALD. A. T. Introdução à Mecânica dos Fluidos. $6^{\mathrm{a}}$ ed. Rio de Janeiro: LTC - Livros Técnicos e Científicos Editora S.A., 2006.

FRIZZARIN, Fernando Bryan. Arduino: Guia para colocar suas ideias em prática. São Paulo: CASA DO CÓDIGO, 2016.

GIMENEZ, Salvador P. Microcontroladores 8051. Editora PEARSON. São Paulo: PEARSON, 2005.

GOMES, M.; ANDRADE, M.; FERRAZ, F. Apostila Hidráulica. Santo Amaro - BA: Centro Federal de Educação Tecnológica da Bahia, 2008. p. 11-28

MERCADO LIVRE, Bombas de diafragma, 2020. Disponível em: https://lista.mercadolivre.com.br/. Acesso em: 11 jul. 2020.

NETTO, José. Pequenas histórias das bombas hidráulicas. Disponível em: http://revistadae.com.br/artigos/artigo_edicao_154_n_66.pdf. Acesso em: 13 jul. 2020.

PINTO, M. Bombas centrífugas - MATERIAL SOBRE CENTRIFUGAS DO CEFET-BA. Disponível em: https://www.docsity.com/pt/bombas-centrifugas-14/4732909/. Acesso em: 15 jul. 2020.

QUEIROZ, Wagner Rodrigues de Oliveira; SOUSA, Wanderson Quaresma. A importância da Plataforma Arduino no meio acadêmico. Revista Científica Multidisciplinar Núcleo do Conhecimento, ano 3, ed. 8, vol. 12, p. 123-133, agosto de 2018.

SLIDEPLAYER, Blog. Área de concentração: contextualização e relação com linhas de pesquisas, 2019. Disponível em: https://slideplayer.com.br/slide/1848270/. Acesso em: 16 jul. 2020.

SOUZA, Pedro H. Apresentação dos cálculos para seleção de bombas de bombas para sistema de reaproveitamento de água de poços artesianos. 2014. Disponível em: 
http://monografias.poli.ufrj.br/monografias/monopoli10012492.pdf. Acessado em: 16 jul. 2020.

TERRON, Luiz Roberto. Operações unitárias para químicos, farmacêuticos e engenheiros: fundamentos e operações unitárias do escoamento do fluido. Rio de Janeiro: LTC, 2012. 